\title{
Recent flow and femtoscopy results from STAR
}

\author{
Grigory Nigmatkulov ${ }^{1, *}$ (for the STAR Collaboration) \\ ${ }^{1}$ National Research Nuclear University MEPhI, 115409, Kashirskoe shosse 31, Moscow, Russia
}

\begin{abstract}
Azimuthal anisotropy and femtoscopic measurements provide information about collectivity and dynamics of the created medium. Data from the BES enables STAR to search for the turn-on/off signatures of the Quark-Gluon Plasma (QGP), for the first-order phase transition, and to constrain various models. In midcentral collisions, the proton and net-proton slope parameter $\left(d v_{1} /\left.d y\right|_{y=0}\right)$ shows a minimum between $\sqrt{s_{N N}}=11.5$ and $19.6 \mathrm{GeV}$. The pion and kaon femtoscopic radii are also measured as a function of transverse mass $\left(m_{T}\right)$, centrality and collision energy, and showing that the system at high energies lives longer.
\end{abstract}

\section{Introduction}

Lattice QCD calculations suggest that the transition from hadronic matter to a Quark-Gluon Plasma (QGP) phase [1] in gold ion collisions at the top energy, where the baryon chemical potential $\mu_{B} \approx 0 \mathrm{MeV}$, of the Relativistic Heavy Ion Collider $\left(\sqrt{s_{N N}} \approx 200 \mathrm{GeV}\right.$ ) is a smooth crossover [2]. At lower energies (higher $\mu_{B}$ ) it was predicted that the transition between these phases may be of the first order [3]. At even higher $\mu_{B}$, the excited nuclear matter is expected to remain in a hadronic phase throughout the interaction. In the scenario of nuclear collisions at the beam energy when the first-order phase transition takes place, various models predict characteristic azimuthal anisotropy signals as well as the time delays of the particle emission. Thus, the study of the azimuthal anisotropic flow and femtoscopic radii in relativistic heavyion collisions will contribute to the exploration of the medium properties.

\section{Data Analysis}

Data sets of $\mathrm{Au}+\mathrm{Au}$ collisions recorded by the STAR Collaboration at $\sqrt{s_{N N}}=7.7,11.5$, 14.5, 19.6, 27, 39, 62.4, and $200 \mathrm{GeV}$ were used in this study. The STAR Time Projection Chamber (TPC) [4] was used to reconstruct particles. Particle identification was performed using the combination of ionization energy loss in the TPC and time-of-flight information from the Time-of-Flight (TOF) detector [5].

\section{Directed Flow}

In high-energy heavy-ion collisions, particles are produced with an azimuthally anisotropic momentum distribution, which is a result of hydrodynamical flow of the QGP. The azimuthal

\footnotetext{
*e-mail: ganigmatkulov@mephi.ru;nigmatkulov@gmail.com
} 
distribution of emitted particles relative to so-called flow symmetry planes (event planes) is typically characterized by its Fourier coefficients $\left(v_{n}\right)$ :

$$
\frac{d N}{d \phi} \propto 1+2 \sum_{n=1}^{\infty} v_{n} \cos \left[n\left(\phi-\Psi_{n}\right)\right],
$$

where $\phi$ is the azimuthal angle of a produced particle, and $\Psi_{n}$ is the azimuthal angle of the $n^{\text {th }}$-order event plane [6].

Rapidity-odd directed flow, $v_{1}^{\text {odd }}(y)$, is the first harmonic coefficient in the Fourier expansion of the final-state azimuthal distribution relative to the reaction plane, and describes a collective sideward motion of emitted particles. The rapidity-even [7] component $v_{1}^{\text {even }}(y)$ is not related to the reaction plane in collisions, and originates from event-by-event fluctuations in the initial nuclei. In these proceedings, $v_{1}(y)$ refers to the odd component.

Hydrodynamic calculations [3, 4] propose the presence of a minimum in net-baryon directed flow versus $\sqrt{s_{N N}}$ as a signature of a first-order phase transition between hadronic matter and QGP. This minimum is related to the softening of the equation of state (EOS). Fig. 1(left,a) shows $d v_{1} / d y$ slope measurements for $\left.\pi^{ \pm}, K^{ \pm}, K_{s}^{0}, \phi, \mathrm{p}, \bar{p}\right), \Lambda$ and $\bar{\Lambda}$ in $\mathrm{Au}+\mathrm{Au}$ collisions at $\sqrt{s_{N N}}=7.7,11.5,14.5,19.6,27,39,62.4$, and $200 \mathrm{GeV}$ [8]. Net protons show a minimum in $d v_{1} / d y$ in the energy range between $\sqrt{s_{N N}}=11.5$ and $19.6 \mathrm{GeV}$ (Fig. 1(left,b)).
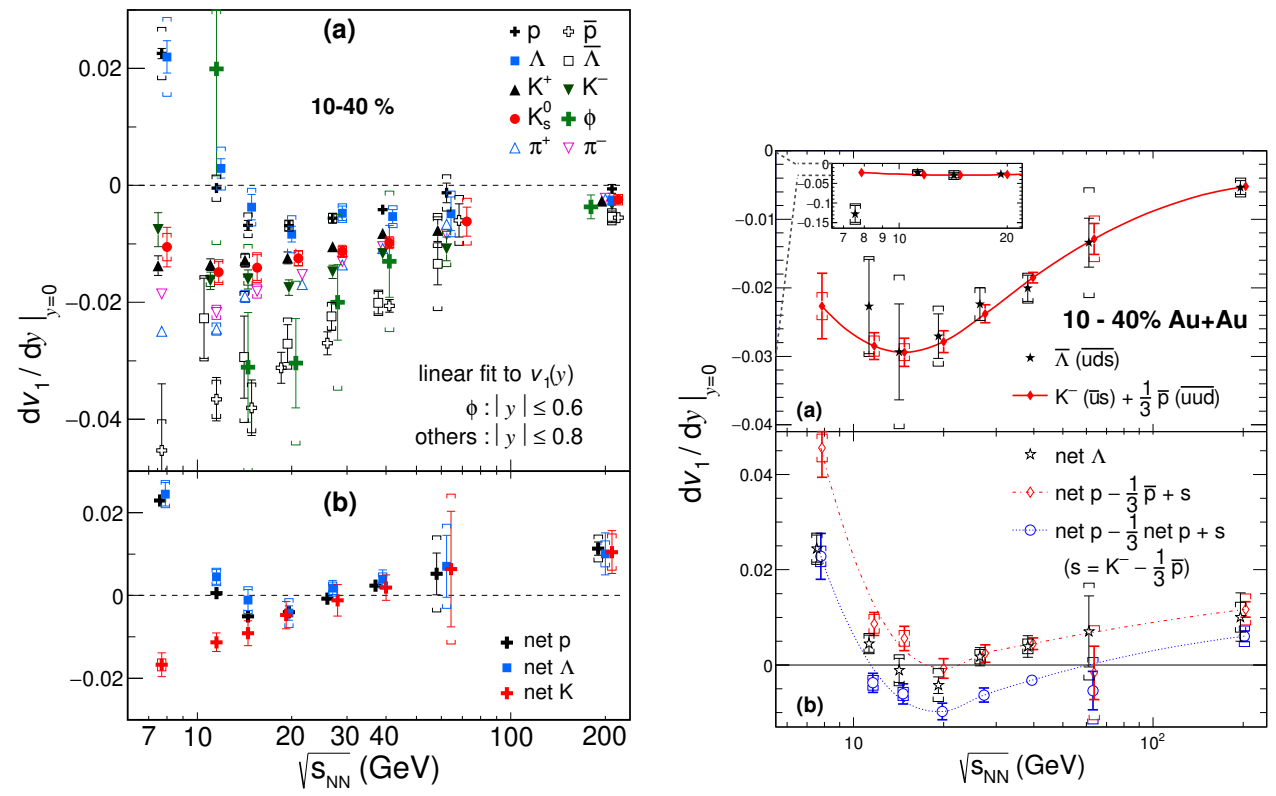

Figure 1. (Left) Directed flow slope $\left(d v_{1} / d y\right)$ versus beam energy for midcentral (10-40\%) $\mathrm{Au}+\mathrm{Au}$ collisions. Panel (a) presents (anti)protons, (anti)Lambdas, $\phi$ meson, and kaons. Panel (b) shows net protons, net $\Lambda$ s and net kaons. (Right) Directed flow slope $\left(d v_{1} / d y\right)$ versus $\sqrt{s_{N N}}$ for midcentral (10$40 \%$ ) collisions. Panel (a) compares the observed $\bar{\Lambda}$ slope with the expectation from the coalescence sum rule for produced quarks. Panel (b) shows test of the coalescence sum rule for net- $\Lambda$ measurement

The ten particle species were used for the investigation of the scaling behavior of $v_{1}$ at constituent quark level versus collision energy [8]. In the test several assumptions have been made. First, $v_{1}$ is developed at the pre-hadronic stage. Second, specific quark types 
have the same directed flow. Third, the registered hadrons are formed via coalescence. In a scenario when deconfined quarks acquire azimuthal anisotropy, and in the limit of small $v_{n}$, coalescence leads to the azimuthal anisotropy of the resulting mesons or baryons being the summed $v_{n}$ of their constituent quarks. We call this the coalescence sum rule.

Firstly, the coalescence sum rule is tested in a scenario when all quarks are known to be produced. Fig. 1(right,a) shows a comparison of the observed $d v_{1} / d y$ for $\bar{\Lambda}(\bar{u} \bar{d} \bar{s})$ with the calculation for $K^{-}(\bar{u} s)+\frac{1}{3} \bar{p}(\bar{u} \bar{u} \bar{d})$. This calculation is based on the coalescence sum rule combined with the assumption that $\bar{u}$ and $\bar{d}$ have the same flow, and that $s$ and $\bar{s}$ have the same flow. A good agreement has been found for the energy range from 200 to $11.5 \mathrm{GeV}$, while a breakdown of agreement was found at $\sqrt{s_{N N}}=7.7 \mathrm{GeV}$ (inset in Fig. 1(right,a)). It implies that one or more assumptions are no longer held below $11.5 \mathrm{GeV}$.

In the limit of low $\sqrt{s_{N N}}$, most $\mathrm{u}$ and d quarks are presumably transported, whereas in the limit of high $\sqrt{s_{N N}}$, most of $\mathrm{u}$ and $\mathrm{d}$ are produced. In Fig. 1(right,b), we exploit net $\Lambda$ (uds) to test two coalescence sum rule scenarios which are expected to bracket the observed $d v_{1} / d y$ for a baryon containing transported quarks.

The first compared calculation (red diamond markers) consists of net proton (uud) minus $\bar{u}$ plus s, where $\bar{u}$ is estimated from $\frac{1}{3} \bar{p}$, and the s quark flow is obtained from $K^{-}(\bar{u} s)-$ $\frac{1}{3} \bar{p}(\bar{u} \bar{u} \bar{d})$. Here we assume that a produced u quark in net $\mathrm{p}$ is replaced with an s quark. This sum-rule calculation agrees closely with the net- $\Lambda$ measurement at $\sqrt{s_{N N}}=19.6 \mathrm{GeV}$ and above, remains moderately close at 14.5 and $11.5 \mathrm{GeV}$, and has a significant deviation at $7.7 \mathrm{GeV}$. The fraction of transported quarks among the constituent quarks of net protons increases with decreasing beam energy, and there is an increasing departure from the assumption that a produced $\mathrm{u}$ quark is removed by keeping the term (net $\left.p-\frac{1}{3} \bar{p}\right)$.

The second coalescence calculation in Fig. 1(right,b) corresponds to $2 / 3$ net proton plus $\mathrm{s}$ (blue circle markers). In this case, we assume that the constituent quarks of net protons are dominated by transported quarks in the limit of low beam energy, and that one of the transported quarks is replaced by s. This approximation seems to hold at $\sqrt{s_{N N}}=7.7 \mathrm{GeV}$, and breaks at higher energies.

\section{Correlation Femtoscopy}

Two-particle interferometry measurement, known as femtoscopy or HBT, is a standard method of studying space-time characteristics of the created system in heavy-ion collisions [9]. It has been most common to perform the femtoscopic analyses with pions [10] as they are the most abundantly produced particles in heavy-ion collisions. The data collected in the BES program allow measurements with the heavier but less abundant particles - kaons. In the current analysis, the same event and pair cuts were used as in [10]. A single-particle selection criteria were extended and in addition to the identification via specific ionization energy loss $(\mathrm{dE} / \mathrm{dx})$ in the TPC, the time-of-flight information from the TOF detector was used to identify pion and kaons. This allowed to perform particle identification in a wide $0.15 \leq p(\mathrm{GeV} / \mathrm{c}) \leq 1.45$ momentum range.

Femtoscopic measurements use correlation function (CF) which in experiments was defined as a ratio of two-particle spectrum (relative momentum, $\mathbf{q}=\mathbf{p}_{\mathbf{1}}-\mathbf{p}_{\mathbf{2}}$ ) from the same event $(N(\mathbf{q}))$ and two-particle spectrum from mixed events $(D(\mathbf{q}))$. In this work, correlation functions were studied in the Bertsch-Pratt coordinate system [11] and $\mathbf{q}$ were decomposed to $q_{\text {out }}, q_{\text {side }}$ and $q_{\text {long }}$ components. The source radii are extracted from the correlation functions by the standard Bowler-Sinyukov [12] method, which is based on the separation of the Coulomb interaction $\left(K\left(q_{\text {inv }}\right)\right)$ from the pure quantum statistics correlation function. To ex- 
tract femtoscopic radii, correlations functions were fitted assuming Gaussian shape of the CF:

$$
C(\mathbf{q})=N\left[(1-\lambda)+\lambda K\left(q_{\text {inv }}\right)\left(1+\exp \left(-R_{\text {out }}^{2} q_{\text {out }}^{2}-R_{\text {side }}^{2} q_{\text {side }}^{2}-R_{\text {long }}^{2} q_{\text {long }}^{2}\right)\right)\right],
$$

where $N$ is normalization factor, $\lambda$ is a correlation strength, $R_{\text {out }}, R_{\text {size }}$ and $R_{\text {long }}$ represent the femtoscopic radii in out, side and long directions, respectively.

Fig. 2 shows the centrality and transverse mass $\left(m_{T}=\sqrt{k_{T}^{2}+m^{2}}\right.$, where $k_{T}=0.5\left(p_{1, T}+\right.$ $\left.p_{2, T}\right)$ ) dependence of the extracted femtoscopic parameters $\lambda, R_{\text {out }}, R_{\text {side }}, R_{\text {long }}$ and $R_{\text {out }} / R_{\text {side }}$ for $\mathrm{Au}+\mathrm{Au}$ collisions at $\sqrt{s_{N N}}=39 \mathrm{GeV}$. Similar measurements have been performed for other collision energies. While the transverse flow and expansion of the system are encoded in the falling of $R_{\text {out }}$ and $R_{\text {side }}$ with increasing $m_{T}$, the longitudinal flow exhibits a decrease of $R_{\text {long }}$ with the increasing $m_{T}$. Such a behavior of source radii $R_{\text {out }}, R_{\text {side }}$ and $R_{\text {long }}$ confirms our expectation that the system under study undergoes space-time evolution and expands. The extracted $R_{\text {out }}$ and $R_{\text {long }}$ values for kaons are generally larger than those for pions at the same transverse mass, while $R_{\text {side }}$ values for pion and kaons are similar.
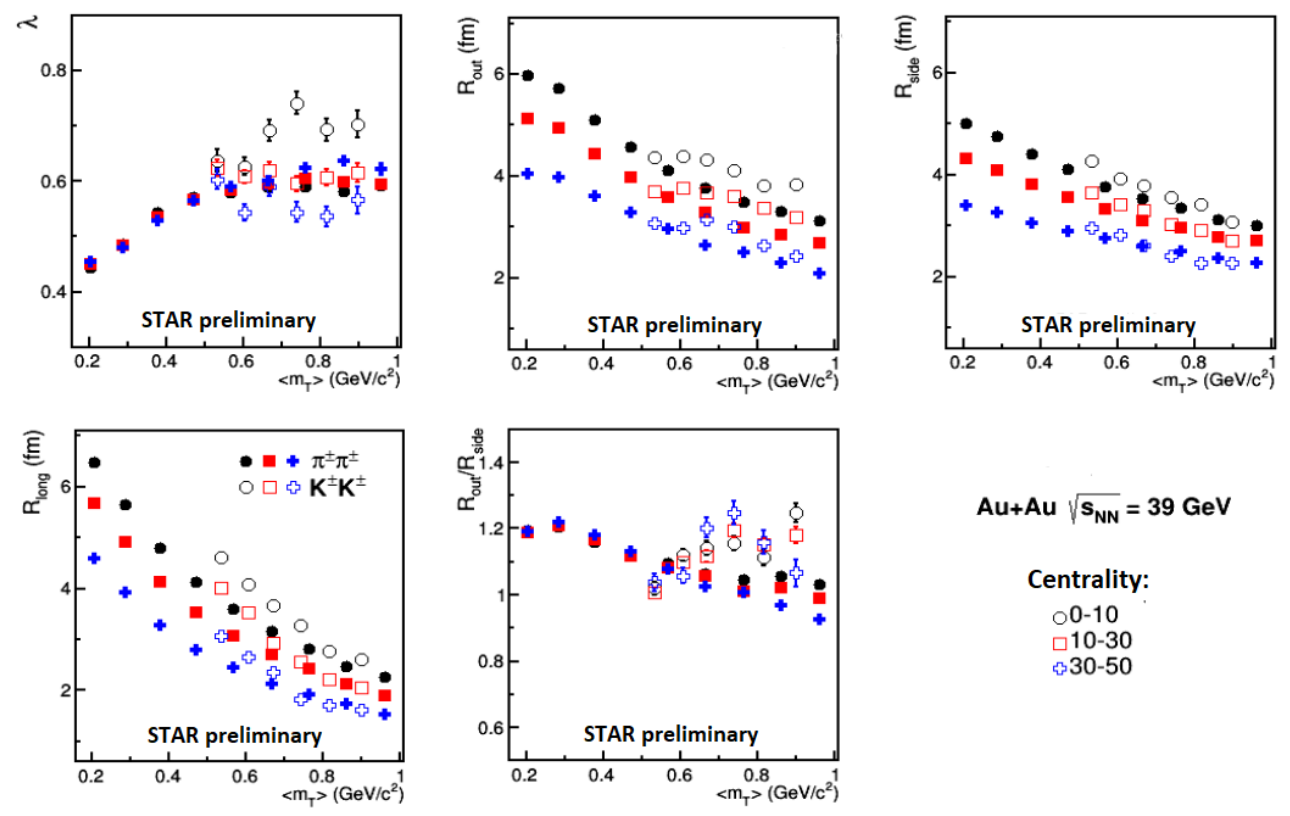

Figure 2. Transverse mass dependence of $\lambda, R_{\text {out }}, R_{\text {side }}, R_{\text {long }}$ and $R_{\text {out }} / R_{\text {side }}$ for pions (solid symbols) and kaons (open symbols) measured for 0-10\% (circles), 10-30\% (squares), and 30-50\% (crosses) most central $\mathrm{Au}+\mathrm{Au}$ collisions at $\sqrt{s_{N N}}=39 \mathrm{GeV}$. Only statistical uncertainties shown

Fig. 3 shows collision energy dependence of the measured femtoscopic radii $R_{\text {out }}, R_{\text {side }}$, $R_{\text {long }}$ for pions (green stars) and kaons (red stars) measured by STAR. Femtoscopic radii $R_{\text {out }}, R_{\text {side }}, R_{\text {long }}$ slightly increase with increasing collision energy up to LHC energy [13] indicating the increase of the expanding source size and lifetime.

\section{Summary}

The results of the directed flow measurements for ten particle species in $\mathrm{Au}+\mathrm{Au}$ collisions at eight beam energies have been presented. These data enable us to test the coalescence 


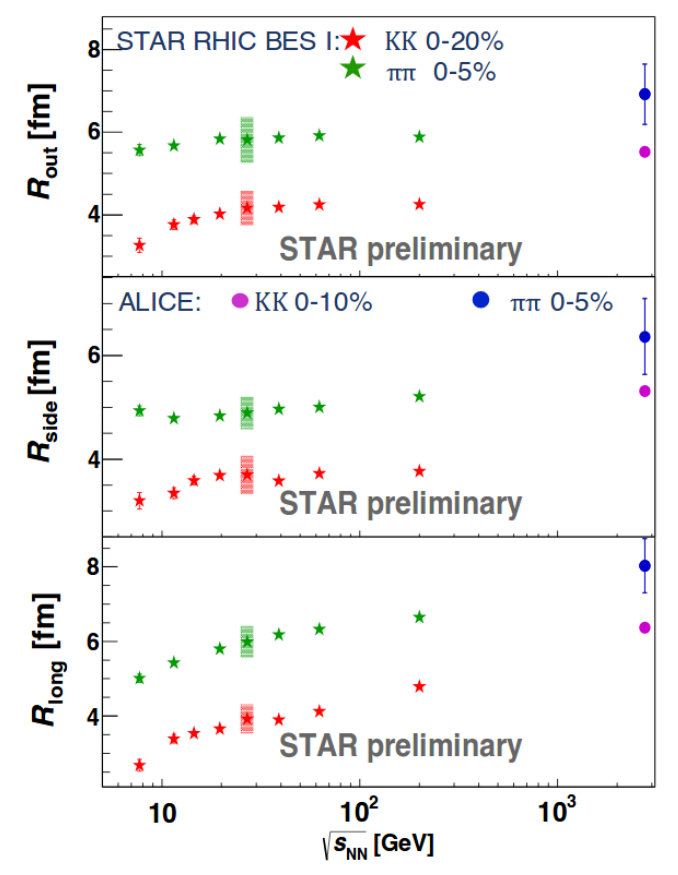

Figure 3. Collision energy dependence of $R_{\text {out }}, R_{\text {side }}, R_{\text {long }}$ for pions (green markers) and kaons (red markers). Stars and circles represent data obtained by the STAR and ALICE experiments, respectively

sum rule on the constituent quark level, for both produced and transported quarks. Two coalescence sum rule scenarios have been explored to bracket the observed $d v_{1} / d y$ of net $\Lambda$ that contains transported quarks. Assuming the validity of the coalescence picture, we extracted the $v_{1}$ slopes of produced $u(\bar{u}, d$ and $\bar{d}), s$ and $\bar{s}$ quarks, as well as transported $u(d)$ quarks as a function of $\sqrt{s_{N N}}$ for midcentral $\mathrm{Au}+\mathrm{Au}$ collisions. Measured femtoscopic radii for identical pion and identical kaon pairs increase with increasing collision energy indicating that the system at high energies lives longer.

Acknowledgements. The reported study was funded by RFBR according to the research project No. 16-02-01119 a, by the National Research Nuclear University MEPhI in the framework of the Russian Academic Excellence Project (contract No. 02.a03.21.0005, 27.08.2013), and by the Ministry of Science and Education of the Russian Federation, grant N 3.3380.2017/4.6.

\section{References}

[1] F. Karsch, Nucl. Phys. A 783, 13 (2007)

[2] F. Karsch et al., Nucl. Phys. B Proc. Suppl. 129, 614 (2004). Y. Aoki, G. Endrodi, Z. Fodor, S.D. Katz and K.K. Szabo, Nature 443, 675 (2006)

[3] S. Ejiri, Phys. Rev. D 78, 074507 (2008). E.S. Bowman and J.I. Kapusta, Phys. Rev. C 79, $015202(2009)$

[4] M. Anderson et al. (STAR Collaboration), Nucl. Instrum. Methods A 499, 659 (2003).

K.H. Ackermann et al., Nucl. Phys. A 661, 681 (1999)

[5] W.J. Llope, Nucl. Instrum. Meth. B 241, 306 (2005) 
[6] S. Voloshin and Y. Zhang, Z. Phys. C 70, 665 (1996). A. M. Poskanzer and S. A. Voloshin, Phys. Rev. C 58, 1671 (1998)

[7] D. Teaney and L. Yan, Phys. Rev. C 83, 064904 (2011). M. Luzum and J.-Y. Ollitrault, Phys. Rev. Lett. 106, 102301 (2011)

[8] L. Adamczyk et al. (STAR Collaboration), Phys. Rev. Lett. 112, 162301 (2014). L. Adamczyk et al. (STAR Collaboration), Phys. Rev. Lett. 120, 062301 (2018)

[9] M.A. Lisa, S. Pratt, R. Soltz, U. Wiedemann, Ann. Rev. Nucl. Part. Sci. 55, 357 (2005)

[10] J. Adams et al. (STAR Collaboration), Phys. Rev. C 71, 044906 (2005). L. Adamczyk et al. (STAR Collaboration), Phys. Rev. C 92, 014904 (2015)

[11] S. Pratt. Phys. Rev. D 33, 1314 (1986). G. Bertsch, M. Gong, and M. Tohyama. Phys. Rev. C 37, 1896 (1988)

[12] M.G. Bowler, Phys. Lett. B 270, 69 (1991). Yu. Sinyukov, R. Lednicky, S.V. Akkelin, J. Pluta, B. Erazmus, Phys. Lett. B 432, 248 (1998)

[13] S. Acharya et al. (ALICE Collaboration), Phys. Rev. C 96, 064613 (2017) 